\title{
Fruit walls and nut shells as an inspiration for the design of bio-inspired impact resistant hierarchically structured materials
}

\author{
R. Seidel ${ }^{1}$, M. Thielen ${ }^{1}$, C. Schmitt ${ }^{1}$, A. Bührig-Polaczek ${ }^{2}$, \\ C. Fleck ${ }^{3} \&$ T. Speck ${ }^{1,4}$ \\ ${ }^{1}$ Plant Biomechanics Group Freiburg, Botanic Garden, \\ Faculty of Biology, University of Freiburg, Germany \\ ${ }^{2}$ Foundry-Institute of the RWTH Aachen, Germany \\ ${ }^{3}$ Materials Engineering, Berlin Institute of Technology, Germany \\ ${ }^{4}$ Competence Networks Biomimetics and BIOKON, Germany
}

\begin{abstract}
Until today the structuring of different types of fruit walls has only been used as an inspiration for packaging when seen from a biomimetic perspective. However, by detailed investigation of the Macadamia nut with its tough testa, Citrus maxima, which possesses a large spongy mesocarp and Cocos nucifera, which has a combination of a fibrous mesocarp and a tough endocarp, it becomes evident that those structures also provide excellent biological role models for impact and puncture resistant materials. Both Citrus maxima and Cocos nucifera are relatively heavy, lack any aerodynamic adaptation and share the same challenge of having to withstand impact from heights of more than 10 metres. By conducting high speed camera controlled free fall experiments of Citrus maxima from six metres high we could demonstrate a deceleration of the fruits of $3100 \mathrm{~m} / \mathrm{s}^{2}$, which corresponds to $316 \mathrm{~g}$, without any visible damage to the fruit. An analysis using cyclic quasi static compression tests of the pericarp of Citrus maxima revealed that the material behaves constantly in good approximation after the first loading cycle. During the first cycle almost $75 \%$ of the energy is dissipated. The pericarp of Citrus maxima is highly visco-elastic, which causes the samples within one minute to recover $30 \%$ of their initial deformation caused by loading to $40 \%$ strain. The mesocarp of Citrus maxima is best described as an open pore foam with gradual increase in pore size. Understanding the principles
\end{abstract}


of how combining structure and material in biological constructions yields a fully functional protection layer will allow us to construct new lightweight bio-inspired materials of high impact and puncture resistance with a combination of high energy dissipation, benign failure and almost complete recovery from large deformations.

Keywords: energy dissipation, biomimetics, citrus maxima, cocos nucifera, Macadamia sp., gradual open pore foam.

\section{Introduction}

The fruit wall, the pericarp, is composed of three layers in all angiosperms. The outmost layer is formed by the exocarp, followed by the mesocarp and the inner most layer, named the endocarp. The pericarp of nuts dehydrates completely and forms a highly lignified, mechanically very stable compact shell. In the case of drupes, only the endocarp lignifies and thereby forms a tough protective layer surrounding the seed. The tough outer layer of the Macadamia presents a special case. The Macadamia 'nut' does not belong to nuts from a botanical point of view as the tough shell is the testa, the seed coat, and not the pericarp. However, the testa of Macadamia sp. is also highly lignified and presents a perfect example for a puncture resistant material [1]. It is made up of two distinct layers where the outer, thicker portion is a very hard sclerenchymatous tissue consisting of fibres and stone cells [1]. Jennings and MacMillan [2] and Wang and Mai $[3,4]$ analysed the biomechanical properties of Macadamia nuts (Macadamia sp.) and came to the conclusion that the structure of the testa ought to be highly optimised for toughness. When compared to ceramic and glass the testa even outperforms these technical materials if the low density of the Macadamia is taken into account. Research on nuts include Balanites aegyptiaca [5], Pistacia vera [6] and roasted shells of cashew nuts, Anacardium occidentale [7], although the focus of these papers is mainly the relationship between the state of hydration and mechanical behaviour in regards to processing in food industries. Mainly compression tests were used and the orientation of the tested nuts was varied to find the lowest energy necessary to crack the shell, which is best achieved at higher velocities $(500 \mathrm{~mm} / \mathrm{min})$.

The coconut, Cocos nucifera, is well known for the potential of its drupes to withstand long exposure to salt water. The drupes are reported to be able to germinate even after a journey of more than $4500 \mathrm{~km}$ in salt water [8]. The fibrous mesocarp of the drupe has mainly been analysed in its dry state for its mechanical properties, as coir fibres are used in carpets, ropes [9], and cushions and seat covers in the automotive industry [10]. The Young's modulus of single dried fibre bundles lies in the range of 3-6GPa and the strains in the range of $15-47 \%$ when tested in tension $[10,11]$. The importance of the entire hydrated mesocarp as a potential material to withstand impact has so far not been addressed, although it is known that the coconut palm reaches up to $30 \mathrm{~m} \mathrm{[7],}$ which equals the potential energy of a $5 \mathrm{~kg}$ fruit of almost $1.5 \mathrm{~kJ}$. Of the genus Citrus only oranges and grapefruits have been studied mechanically to some extent. Fluck and Ahmed [12] examined the degree of internal damage as 
inflicted on the fruits by single and repeated dropping from various heights of up to one meter. By conducting a compression test afterwards it was revealed if a fruit had experienced impact before. Underhill et al. [13] analysed the dynamic and static compressive behaviour of "Eureka" lemons. Their morphological analyses showed that the albedo is compacted during dynamic testing and ruptures during static testing. In a first report Seidel et al. [14] analysed the mechanical properties of Citrus maxima. According to their study the fruits can withstand free falls from up to $15 \mathrm{~m}$ onto wet grass without major damage. Free fall experiments onto a PMMA plate from $6 \mathrm{~m}$ yielded impact durations from
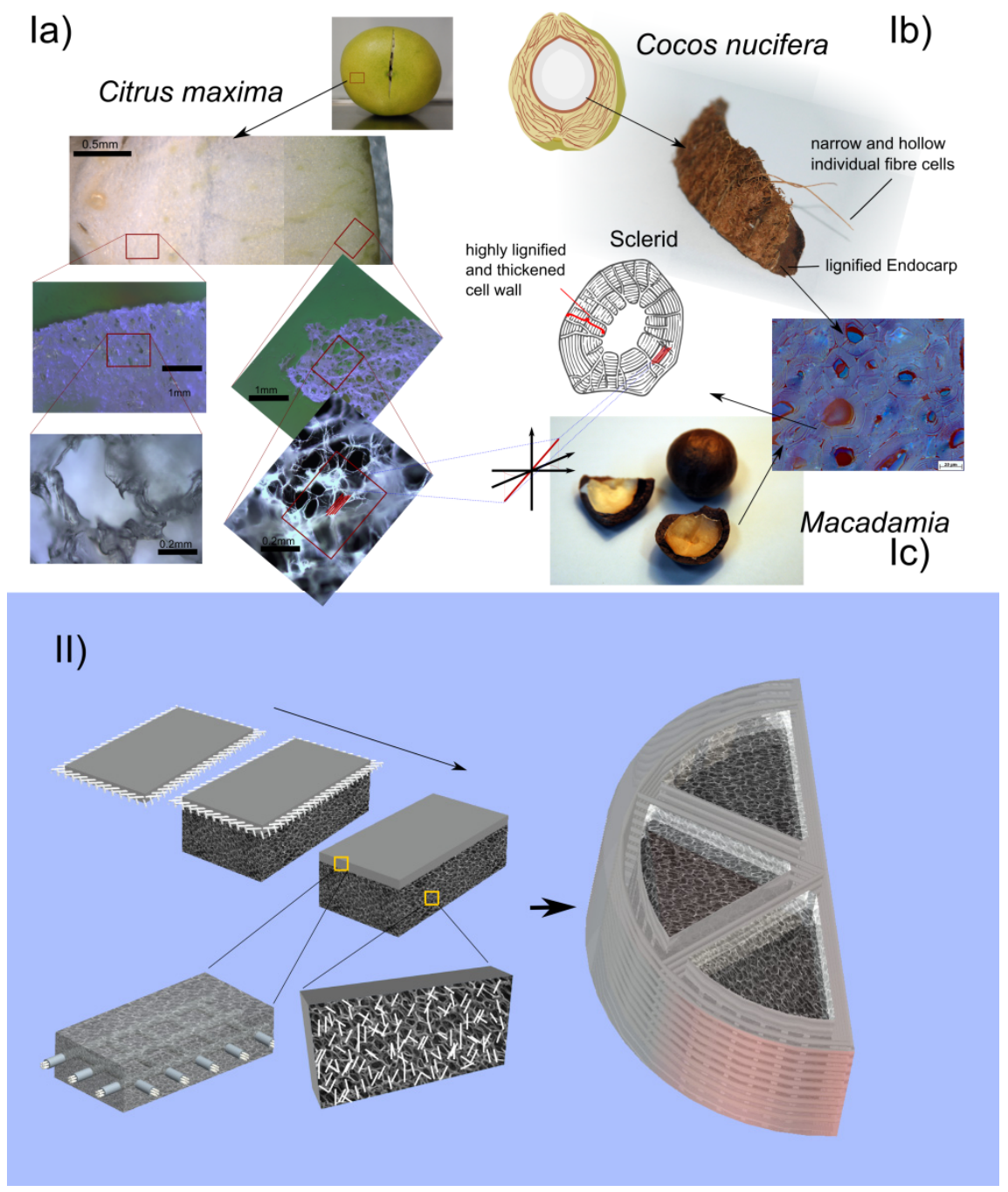

Figure 1: Biological role models and technical implementation. 
approximately $7 \mathrm{~ms}$, forces of $1 \mathrm{kN}$ and a total dissipation of $90 \%$ of the initial potential energy. Their morphological analysis indicated a gradual increase in intercellular space from the exocarp to the endocarp, although the anatomy was only examined with a stereo microscope.

In summary it may be said that research on fruit walls so far has mostly focused on mechanical properties important to the food and fibre industries and little has been done on the form-structure-function-relationship, which has been hardly studied at all in Citrus maxima, and only to some extend in Cocos nucifera and Macadamia sp., although all of these fruit walls reveal quite extraordinary mechanical properties. Further, no related studies on impact and energy dissipation during impact, which will be mostly achieved by the deformation of the mesocarp structure in drupes and Citrus, are available.

As shown in Figure 1 the hierarchically organised fruit walls of our role models compose either (Ia) a highly damping spongy gradient structure (Citrus maxima), (Ib) a combination of a fibrous damping structure with a tough shell (Cocos nucifera) or (Ic) an entire tough shell (Macadamia sp.). These structural elements will be transferred into hierarchically organized technical materials (II) consisting of a solid layer in combination with a spongy fibre reinforced structure ensuring high toughness and high energy dissipation. The following parts of this paper will focus on our results as to the functional morphological and biomechanics of Citrus maxima.

\section{Materials and methods}

\subsection{Dynamic testing}

To analyse the potential of the whole Citrus maxima fruit to absorb energy during impact, free fall experiments were conducted. Single fruits were dropped from six metres onto a PMMA plate, which was allowed to rotate on one side and was freely positioned on a force sensor on the opposite side (Fig. 2, left). The fruits' impact behaviour was filmed using two high speed cameras capturing at 240 and 1000 frames per seconds, respectively. The analysis of the frames from the side view camera allowed an estimation of the deceleration of the fruit at impact.

\subsection{Static testing}

Samples of the pericarp of Citrus maxima were cut with a cylindrical cork knife and tested in compression using an Instron testing machine (testing speed $5 \mathrm{~mm} / \mathrm{min}$ ). All samples were cut along the radial axes of the fruit. To examine the energy absorption and the potential to absorb energy in consecutive cycles four compression cycles to $40 \%, 40 \%, 40 \%$, and $75 \%$ compression, respectively, were conducted. Between the cycles the sample was allowed to relax for 60 seconds. 


\subsection{Morphology}

The pericarp of Citrus maxima was cut into semi-thin sections of about $2 \mathrm{~mm}$ thickness each at different positions on the fruit. The sections were immediately analysed using advanced light microscopy to prevent any artefacts due to dehydration of the tissue. A second set of sections was embedded in a PEG 2000, water solution $(1 / 1)$ and left for 4 days at $55^{\circ} \mathrm{C}$ for the water to evaporate. The sections where then cut with a freezing microtome to $10 \mu \mathrm{m}$ thick subsections and stained with Toluidin blue (Fig. 4). To analyse the distribution of cell wall, cell lumen and intercellular space, the slice was photographed and the digital image processed with GIMP (version 2.6) to separate the materials and set to a single colour value, blue, green, and red, respectively.

\section{Results and discussion}

To estimate the deceleration of the whole fruit during impact the side view camera of the free fall experiments presented by Seidel et al. [14] were analysed frame by frame. The capture rate of the camera with $240 \mathrm{fps}$ was too slow to yield sharp images of the fruit at each time-point; though due to the blurred position an estimate was possible about the action within each frame of $3.5 \mathrm{~ms}$ time duration when approximating the time for the shutter to open and close with $\sim 0.66 \mathrm{~ms}$. Figure 2 presents the case of the fruit immediately before impact and during impact just before initiating the bouncing off the plate again. The initial speed

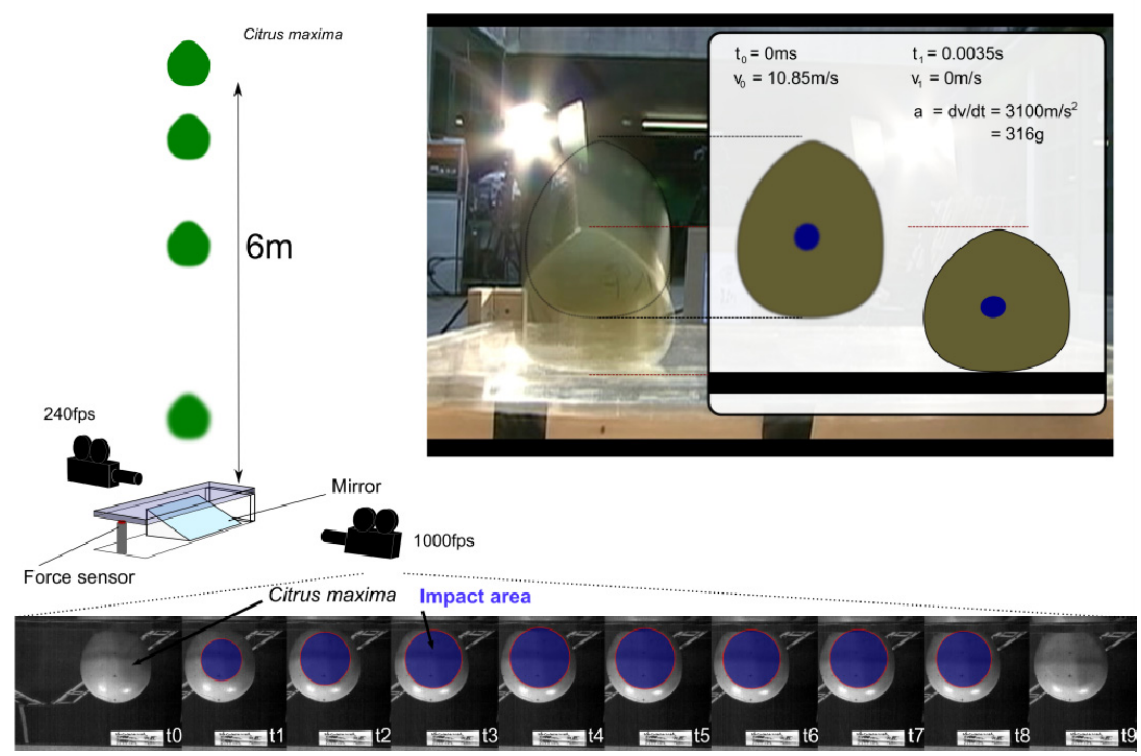

Figure 2: $\quad$ Free fall experiment of Citrus maxima. 
equals the full conversion of potential energy into kinetic energy and calculates to $10.85 \mathrm{~m} / \mathrm{s}$. Before it bounces off the speed will be $0 \mathrm{~m} / \mathrm{s}$. Dividing the change in speed by the time duration of the frame yields a deceleration of $3100 \mathrm{~m} / \mathrm{s}^{2}$, which corresponds to $316 \mathrm{~g}$. Multiplying this by an average fruit weight of about $1.5 \mathrm{~kg}$ results in forces of about $4.6 \mathrm{kN}$ acting on the fruit. The calculated forces are about 4.5 times higher than reported [14], which is most likely due to the sample rate of the force sensor in combination with the amplifier used in their setup. As at the beginning of the $3.5 \mathrm{~ms}$ of the frame duration the fruit is not in contact with the plate, the time duration of the impact may be even lower, resulting in higher decelerations. Seidel et al. [14] reported impact durations of the same setup of $7.2 \mathrm{~ms}$. Assuming that this time is split evenly between deceleration and acceleration, results in $3.6 \mathrm{~ms}$ per event, which is very close to the approximated $3.5 \mathrm{~ms}$ in the calculation presented above.

Consecutive static compression tests of the pericarp of Citrus maxima to $40 \%$ compression confirmed a loss of maximum $75 \%$ energy within one hysteretic cycle [14]. Further from the second cycle onwards the material behaviour remains in good approximation constant as shown in Figure 3 (upper diagram). As there was one minute pause between the cycles one can distinguish at least partly between plastic and visco-elastic deformation. During this minute the material recovers its shape from circa $22 \%$ compression, the compression at which the unloading curve yields 0 force, to about $15 \%$ compression, the compression at which the force rises above zero in the next loading curve. These $7 \%$ gain in shape can be fully accounted to the visco-elastic properties of the material. In the final compressive loading the material is loaded beyond the onset strain of material densification. The onset strain of densification is approximately at the strain where the tangent to the compression curve intersects the abscissa (Fig. 3, lower diagram). In the case of Citrus maxima the onset strain of densification varies between $55 \%$ to $65 \%$ strain.

As reported by Seidel et al. [14] the fruit wall of Citrus maxima comprises three distinguishable parts, the exocarp containing multiple compact layers of cells, the mesocarp, showing a spongy appearance, and the endocarp consisting of a small dense layer adjacent to the pulp. The analysis of thin sections allowed one to quantify the material distribution of cell wall material, cell lumen, and intercellular space. The two lower graphs in Figure 4 show a continuous increase in the intercellular space from the innermost part of the exocarp towards the middle of the mesocarp. This is in accordance with the reported reduction in cell density and the beginning of cavity formation composing a three dimensional porous spongy tissue [14]. The cell wall material continuously decreases from $80 \%$ to $40 \%$ contribution to the cross-sectional area within the first millimetre, which presents the overlap between exo- and mesocarp. It remains at $40 \%$ contribution to the cross-sectional area until about $4 \mathrm{~mm}$ before it starts to oscillate between $25 \%$ and $35 \%$ area fraction. The lumen almost constantly vary between $15 \%$ and $30 \%$ contribution to the cross-sectional area but decrease from the $8^{\text {th }}$ millimetre onwards to less than $20 \%$. The most prominent result of this analysis is the proof of the predicted increase in intercellular space [14]. Within the first $300 \mu \mathrm{m}$ of within the exocarp no intercellular space is found, 
Compression tests ( $40 \%$ strain)

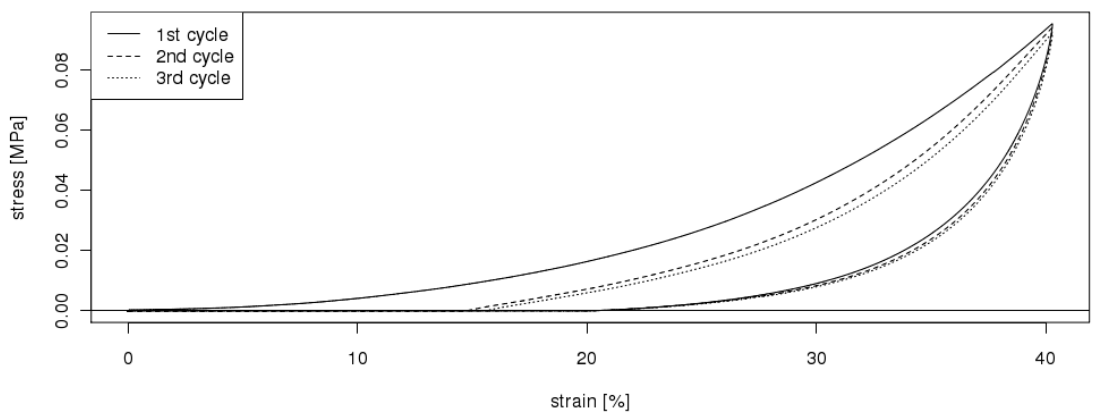

Compression tests $(75 \%$ strain)

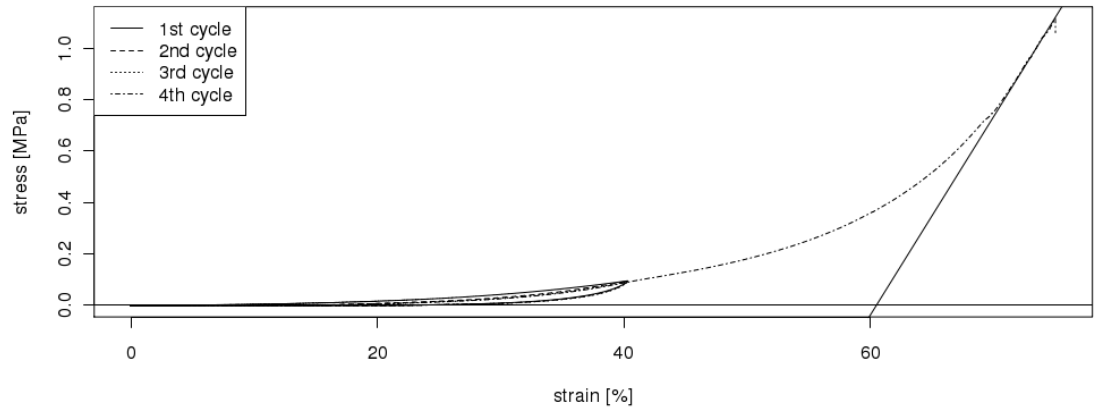

Figure 3: $\quad$ Static compression tests of Citrus maxima.

thereafter its contribution to the cross-sectional area rises quickly and reaches $30 \%$ at the $1^{\text {st }}$ millimetre. Afterwards the portion of the intercellular space remains rather constant until about 4 millimetres before it rises again to values up to $80 \%$ area fraction.

The most important features of Cocos nucifera with regard to its potential as a biological role model for an impact and puncture resistant bio-inspired material are from a morphological point of view the sandwich structure of a high energy absorption layer, its mesocarp, and a tough puncture resistant layer, its highly lignified endocarp. The fibres and fibre bundles embedded in the mesocarp show benign failure only at $30 \%$ to $40 \%$ strain [14], which is a very desirable property for a technical material. As reported by Wang and Mai [4] the Macadamia testa is highly optimised for toughness. Properties very similar to the Macadamia can be predicted for the tough endocarp of the coconut as well, as it has a very similar structure being composed of a very similar type of highly lignified cells.

Combining these extraordinary protective properties of high energy dissipation, benign failure behaviour and almost full recovery from large deformations as it is seen in Citrus maxima after the first loading cycle will lead to new bio-inspired light-weight technical materials. A bio-inspired sandwich material consisting of a high energy absorption outer layer in combination with a 

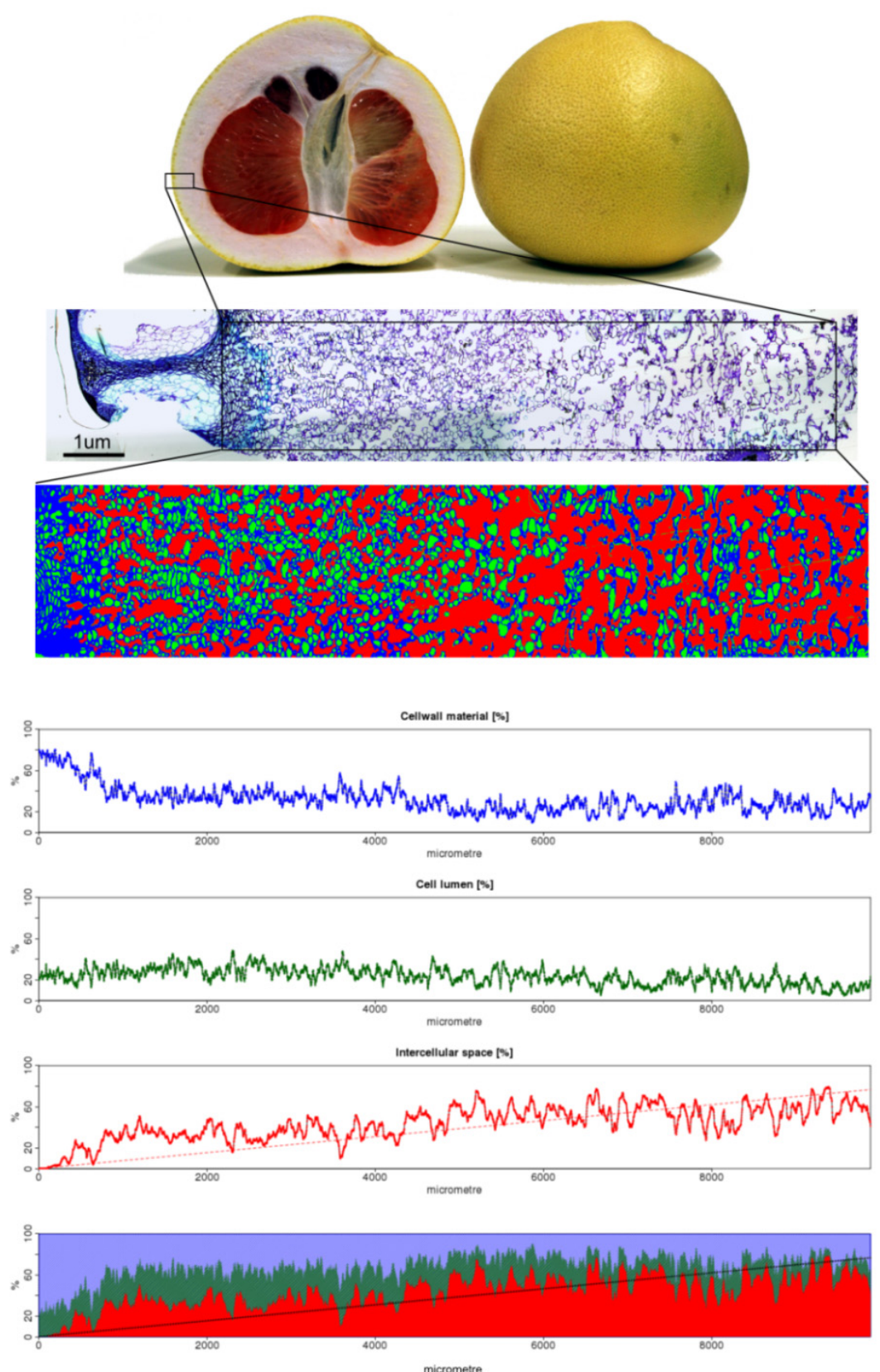

Figure 4: Analysis of the distribution of material within the fruit wall of Citrus maxima. 
very tough puncture resistant inner layer is one of our first objectives. Cocos nucifera is often regarded as one of the best natural role models for man-made impact resistant structures as the fruits have to withstand impacts from more than $25 \mathrm{~m}$, although as impressively shown by Citrus maxima, multiple pathways can be used to achieve high energy dissipation. In their hierarchical structures, biological materials make use of cellular foams, spongy structures, laminates, fibres and various combinations of these structures on different structural levels. Investment casting allows to model open pore sponge structures, as well as the integration of fibres. The transfer of the properties of the biological, organic materials into technical materials like metallic cast alloy and fibre-reinforced composite materials with a matrix made of metal, glass, ceramics or various polymers will be one of the major challenges in the technical transfer.

Potential applications for biomimetic impact resistant fibre reinforced foams are vessels for transportation of dangerous goods, e.g. explosives or hydrofluoric acid, helmets and other protection wear, impact protection of vehicles and space station protection against meteoroid impact.

\section{Acknowledgement}

We would like to thank the German Research Foundation for the funding of this project as part of the Priority Program 1420 "Biomimetic Materials Research: Functionality by Hierarchical Structuring of Materials" (SP 534/10-1).

\section{References}

[1] Hartung, M.E. \& Storey, W.B., The development of the fruit of Macadamia ternifolia, Journal of Agricultural Research, 59, pp. 397 - 406, 1939.

[2] Jennings, J.S. \& Macmillan, N.H., A tough nut to crack, Annals of Botany, 46, pp. $313-321,1980$.

[3] Wang, C. \& Mai, Y., Deformation and fracture of Macadamia nuts Part 2: Microstructure and fracture mechanics analysis of nutshell, International Journal of Fracture, 69, pp. $67-85,1995$.

[4] Wang, C. \& Mai, Y., Deformation and fracture of Macadamia nuts Part 1: Deformation analysis of nut-in-shell, International Journal of Fracture, 69, pp. $51-65,1995$.

[5] Mamman, E., Umar, B. and Aviara, N., Effect of Moisture Content and Loading Orientation on the Mechanical Properties of Balanites Aegyptiaca Nuts, Agricultural Engineering International, The CIGR Ejournal 7, Manuscript FP 04 015, 2005.

[6] Ozden, K. \& Alayunt, F., The Determination of Some Physical Properties of Pistachio vera L., Pakistan Journal of Biological Sciences, 9(14), pp. $2612-2617,2006$.

[7] Oloso, A. O. \& Clarke, B., Some aspects of strength properties of Cashew nuts, Journal of agricultural engineering research: 55, 27 - 43, 1993.

[8] Lieberei, R. \& Reisdorff, C., Nutpflanzenkunde, Georg Thieme Verlag, Stuttgart, 2007. 
[9] Varma, D.S., Varma, M. \& Varma, I.K., Coir fibers: Part I: Effect of Physical and Chemical Treatments on Properties, Textile Research Journal, 54, pp. $827-832,1984$.

[10] Goulart Silva, G., De Souza, D.A., Machado, J.C. \& Hourston, D.J., Mechanical and thermal characterization of native Brazilian coir fiber, Journal of Applied Polymer Science, 76, pp. 1197 - 1206, 2000.

[11] Kulkarni, A.G., Satyanarayana, K.G., Sukumaran, K. \& Rohatgi, P.K., Mechanical behaviour of coir fibres under tensile load, Journal of Material Science, 16, pp. 905 - 914, 1981.

[12] Fluck, R.C \& Ahmed, E.M., Measurement by Compression Test of Impact Damage to Citrus Fruits, Journal of Texture Studies, 4, 494 - 500, 1974.

[13] Underhill, S.J.R., Mclauchlan, R.L., Dahler, J.M., Bertram, J., Flavedo and albedo changes in, 'Eureka' lemons caused by static compression and impact loading, Journal of Texture Studies, 29, 437 - 452, 1998.

[14] Seidel, R. Bürig-Polaczek, A., Fleck, C. \& Speck, T., Impact resistance of hierarchically structured fruit walls and nut shells in view of biomimetic applications, Proceedings of the $6^{\text {th }}$ Plan Biomechanics Conference, ed. Thibaut, B., French Guyana, France, ECOFOG, pp. 406 - 411, 2009. 